\title{
Stability Indicating Ion-Pair Reversed-Phase Liquid Chromatography Method for Modified mRNA
}

\author{
Jonathan Currie ${ }^{1}$, Jacob R. Dahlberg ${ }^{2}$, Jonas Eriksson², Fritz Schweikart ${ }^{2}$, Gunilla A. Nilsson ${ }^{2}$ and Eivor Örnskov² \\ ${ }^{1}$ Innovation Strategies and External Liaison, Pharmaceutical Technology and Development, Operations \& IT, AstraZeneca, \\ Gothenburg, Sweden \\ ${ }^{2}$ Advanced Drug Delivery, Pharmaceutical Sciences, BioPharmaceuticals R\&D, AstraZeneca, Gothenburg, Sweden
}

\begin{abstract}
Modified messenger RNA (mRNA) represents a promising new class of therapeutic drug product. Development of robust stability indicating methods, for control of product quality, are therefore critical to support successful pharmaceutical development. This paper presents the first ion pair reversed phase liquid chromatography (IP-RPLC) method to characterise modified mRNA exposed to a wide set of stress-inducing conditions, relevant for pharmaceutical development of an mRNA drug product. The optimised method could be used for separation and analysis of large RNA, sized up to 1000 nucleotides. The impacts of column temperature, mobile phase flow rate and ion-pair selection were studied and optimised. Baseline separations of the model RNA ladder sample were achieved using all examined ion-pairing agents. We established that the optimised method, using $100 \mathrm{mM}$ Triethylamine, enabled the highest resolution separations for the largest fragments in the RNA ladder (750/1000 nucleotides), in addition to the highest overall resolution for the selected modified mRNA compound (eGFP mRNA, 996 nucleotides). The stability indicating power of the method was demonstrated by analysing the modified eGFP mRNA, upon direct exposure to heat, hydrolytic conditions and treatment with ribonucleases. Our results showed that the resulting degradation products, which appeared as shorter RNA fragments in front of the main peak, could be well visualized, using the optimised method, and the relative stability of the mRNA under the various stressed conditions could be assessed.
\end{abstract}

Keywords: Modified mRNA, Ion Pair Reversed-Phase Liquid Chromatography, Stability indicating, RNA ladder, Ribonucleic acid, Nucleotides, Chemical degradation, Degradation study

\section{Introduction}

Chemically messenger RNA (mRNA) constitutes an emerging new class of drug product, which has shown rapidly increasing potential over recent years. Improvement in intracellular stability and translational efficiency of modified mRNA into therapeutic proteins has led to development of novel drug products that are currently being tested in clinical studies worldwide ${ }^{1,2}$. These modified mRNA compounds are single-stranded polynucleotides, typically containing between 800 and 8000 bases. AstraZeneca has recently reported the successful first in-human study of this modality type with its AZD8601 compound ${ }^{3}$. In addition, the mRNA modality platform has shown excellent potential with which to enable rapid drug-discovery and development. This has been well exemplified in the rapid progression of mRNA-based vaccine therapeutics in the response to the SARS-CoV-2 pandemic ${ }^{4-6}$. The increased focus towards this new type of drug product has created a need for effective and reliable characterisation methods, which can be used during development of mRNA drug products.
Stability studies are critical components of pharmaceutical development processes and require a range of robust and reliable analytical methods. During manufacture and storage of both drug substance and drug product, mRNA can rapidly degrade through exposure to heat, light, hydrolytic and oxidative conditions, as well as through contamination with ribonucleases ${ }^{7}$. For therapeutic use, mRNA is typically modified so it is more resistant to chemical as well as in vivo degradation compared to unmodified mRNA. While processes for in vivo degradation of mRNA have been widely studied, in contrast the chemical degradation pathways relevant for pharmaceutical quality testing are sparsely described, and the number of reported stability indicating methods is low. To validate the stability indicating power of an analytical method, stability testing of samples containing the active pharmaceutical ingredient should be carried out under stress test conditions that are more severe than those used for long term stability studies. The European Medicines Agency, (CPMP/ICH/2736/99) states that "Stress testing of the drug substance can help identify the likely degradation products, which can in turn help establish the degradation pathways and the intrinsic stability of the molecule and validate the stability indicating power of the analytical procedures used. The 
nature of the stress testing will depend on the individual drug substance and the type of drug product involved." It should be noted that due to the novelty of this emerging area, the regulatory requirements for quality control of mRNA drug products are not yet fully established ${ }^{9}$. For mRNA vaccine drug products, the regulatory guidelines for vaccines can be applied, while non-vaccine mRNAbased drug products are currently viewed as gene therapy treatments by regulatory bodies ${ }^{10}$. Modified eGFP mRNA (996 nucleotides) is a compound widely used in the development of various RNA drug delivery systems, owing to the fluorescent properties of its expressed protein.

The utility of Ion Pair Reverse-Phase Liquid Chromatography (IP-RPLC) for separation of large RNA compounds, up to 8000 nucleotides, has been demonstrated ${ }^{11-17}$. Separation of polar mRNA analytes is facilitated by ion pairs that are formed between the negatively charged mRNA backbone and a positively charged ion pairing agent added to the mobile phase. The mRNA-ion pair complex displays an increased affinity to the stationary phase compared to mRNA alone, resulting in increased retention on reverse phase columns. Dickman et al suggests that IP-RPLC can offer the ability to analyse a wide range of RNA compounds through modification of chromatographic conditions, including choice of ionpairing agents, temperature and mobile phase compositions ${ }^{14}$. Commonly used ion-pair combinations for analysis of large RNA include: Triethylammonium Acetate (TEAA), Hexylammonium Acetate (HAA) and Dibutyl ammonium Acetate (DBAA).

Chromatographic separation of high molecular weight RNA has a number of challenges. Slow mass transportation, dispersity of the RNA compounds, and the influence of variable secondary and tertiary RNA structures can all impact the achievable separation. In addition, using IP-RPLC for analysis of large RNA, insufficient column pore sizing can impact separation, restricting mass transport through the column, as well as reducing available surface area for ion-pair interactions. This can limit the ability to optimise method parameters, such as mobile phase gradient, flow rate and composition, to achieve the desired separation ${ }^{15}$. Lloyd et al have shown that large pore sizes (4000§) are typically needed to resolve large nucleotides greater than 500 base pairs. Stationary phases based on polystyrene divinylbenzene (PS-DVB) polymer matrices are available with large pore sizes, and promising separations of RNA compounds and RNA ladders have been demonstrated ${ }^{18}$. Azarani, Georgopoulos, and Dickman et al. have demonstrated and described separation of large RNAs using columns based on alkylated PS-DVB co-polymeric stationary phases, such as DNASep columns from Transgenomic 13, 14, 16 . Yamauchi et al. have presented separations of large RNA ladders up to 8000 nucleotides using a PLRP-S column, from Polymer Labs, which has a stationary phase based on monolithic non-alkylated PS-DVB, with pore size: $4000 \AA$. In their findings, it is suggested that one of the key factors in enabling high resolution separations for large RNA fragments, using IP-RPLC, is selecting an appropriate column pore size for the analyte size of interest ${ }^{11}$.

Additionally, column temperature plays an important role in improving peak resolution during RNA separation analyses. Waghmare et al. have previously demonstrated that high resolution separations of ssRNA, up to 1000 nucleotides, can be achieved in IP-RPLC using high temperature column conditions ${ }^{19}$. Similarly, Roussis et al. report that analysis of oligonucleotides at elevated temperatures, using IP-RPLC, improved impurity resolution ${ }^{20}$. Higher column temperatures are suggested to help facilitate denaturating conditions in the presence of an organic co-solvent (i.e $\mathrm{MeCN})^{21}$. Thus, the impact of secondary/tertiary RNA structures on the analyses can be reduced.

Azarani et al. have previously established that IP-RPLC methods can be utilised to detect and assess RNA transcript degradation ${ }^{13}$. The study reports analysis of a transcription reaction product using IP-RPLC. The results showed that smaller and/or degraded RNA fragments could be well distinguished from the full-length transcript during analysis. More recently, the study by Kanavaroti has demonstrated the most informative approaches to characterisation of large RNA, showing the use of an ion exchange LC (IEX) and an IP-RPLC method to examine RNA fragments, including modified mRNA ${ }^{22}$. The study importantly demonstrated that these types of methods could potentially be exploited for quality control and purity determination purposes. We propose that an IPRPLC method is preferable for stability indication of modified mRNA, in comparison to an IEX method due to the ability to operate at a lower $\mathrm{pH}$. Hydrolysis of mRNA is one of the main degradation pathways and potentially on-column analyte stability is impacted by these conditions. Additionally, the use of IP-RPLC would allow use of mass spectrometry for detection/identification of shorter degradation products ${ }^{23,24}$.

It should also be noted that additional types of mRNA degradation products may require alternative techniques and set-ups to be monitored. For the quantification of small degradation products, screening of additional UV wavelengths could enable sensitivity improvements when compared to the UV detection wavelength typically used in IP-RPLC methods for RNA $(260 \mathrm{~nm})$. The most prominent RNA degradation pathways result in the formation of shorter RNA fragments, however, degradation pathways which result in modification of the nucleotides, for example exposure to light, oxidation, depurination and deamidation require complementary/orthogonal methods for analysis ${ }^{25-29}$. We propose that separation methods in conjunction with MS, MS/MS and relevant bioassays, should be used to fully characterize the stability of mRNA drug products.

To the best of our knowledge, to date, there are few reports describing development of stability indicating methods applicable for pharmaceutical 
development of large mRNAs using

liquid

chromatography. The aim of this study was to identify and evaluate key separation parameters to consider in developing stability indicating IP-RPLC UV methods for mRNA. Various

aspects for developing IP-RPLC

methods of large mRNA are discussed. Baseline separation of all peaks of an RNA ladder comprising of seven fragments $(100,200,300,400,500,750$ and 1000 nucleotides) is demonstrated using optimized IPRPLC method conditions. The stability indicating power of the optimized IP-RPLC method is demonstrated by analysis of eGFP modified mRNA that has undergone stressed degradation.

\section{Experimental}

\section{Materials}

Acetonitrile (99.9\% Purity) and Hydrochloric Acid (37\%) (12 M) were purchased from Merck (Darmstadt, Germany). Triethylamine (TEA) (99.5\% purity), Dibutylamine (DBA) (99.5\% purity), Hexylamine (HA) (99.5\% purity) and Sodium Hydroxide (98\% purity) were all purchased from Sigma-Aldrich (St. Louis, USA). Acetic Acid (glacial) (99.7\% purity) was purchased from Alfa Aesar (Kandel, Germany). Distilled water for mobile phase preparation was prepared through a Millipore MilliQ Direct Ultrapure water system, while RNase-free water for sample preparation, was filtrated through a Millipore Biopak Polisher (Darmstadt, Germany). Ambion Century Plus single-stranded RNA markers (100, 200, 300, 400, 500,750 and 1000 nucleotide fragments) were purchased from Invitrogen ThermoFisher Scientific (Vilnius, Lithuania). The RNA markers were received as $50 \mu \mathrm{L}$ of $1.0 \mathrm{mg} / \mathrm{mL}$ RNA in $0.1 \mathrm{mM}$ EDTA. Clean-Cap enhanced green fluorescent protein (eGFP) modified mRNA (996 nucleotides) with a fully substituted 5-Methoxy-Uridine base composition was purchased from TriLink Biotechnologies (San Diego, USA). The model mRNA sample was delivered as $1 \mathrm{~mL}$ of $1.0 \mathrm{mg} / \mathrm{mL} \mathrm{mRNA}$ in 1 $\mathrm{mM}$ sodium citrate buffer ( $\mathrm{pH} 6.4$ ).

\section{Sample Preparation}

Prior to analysis, mRNA and RNA ladder samples were stored in a freezer at $-80^{\circ} \mathrm{C}$. As required, samples were thawed at room temperature and carefully mixed by repeated pipetting. RNA samples were prepared within a vertical laminar air flow cabinet from ScanLAF A/S (Lynge, Denmark). For samples used to establish suitable separation parameters, the RNA ladder samples were diluted to $0.05 \mathrm{mg} / \mathrm{mL}$ by addition of RNase-free water. For ion pair comparison and stress testing studies, both the RNA ladder samples and the mRNA samples were diluted to $0.5 \mathrm{mg} / \mathrm{mL}$ through addition of RNase free water.

Stress testing of eGFP mRNA was carried out under hydrolytic conditions, treatment with RNase A, and prolonged exposure to high temperature conditions respectively:
High/Low pH: $100 \mu \mathrm{L}$ sample vials were prepared containing $50 \mu \mathrm{L}$ eGFP mRNA at $1.0 \mathrm{mg} / \mathrm{mL}$ and either $50 \mu \mathrm{L}$ of $0.01 \mathrm{M} \mathrm{HCl}$ or $50 \mu \mathrm{L}$ of $0.02 \mathrm{M} \mathrm{NaOH}$, resulting in a solution of $0.5 \mathrm{mg} / \mathrm{mL}$ mRNA in corresponding high or low $\mathrm{pH}$ media. RNase treatment was carried out by adding $5 \mu \mathrm{L}$ of $0.1 \mu \mathrm{g} / \mathrm{mL}$ RNase A solution to a vial containing $50 \mu \mathrm{L} 1.0 \mathrm{mg} / \mathrm{mL}$ eGFP mRNA and $45 \mu \mathrm{L}$ RNase-free water. These samples were stored in the autosampler at $25^{\circ} \mathrm{C}$ and tested at intervals until significant degradation was observed.

Heat: eGFP mRNA $(1.0 \mathrm{mg} / \mathrm{mL})$ was heated at $85^{\circ} \mathrm{C}$ at time intervals up to 240 minutes and immediately placed on ice for 5 minutes. mRNA samples were then diluted to $0.5 \mathrm{mg} / \mathrm{mL}$ by addition of RNase free water before use. Autosampler was kept at $10^{\circ} \mathrm{C}$ to minimise further degradation.

\section{Instrumentation}

All testing was carried out using an Agilent 1200 HPLC system (Santa Clara, USA). The system consisted of a binary solvent pump with degasser unit, a G1315C UV diode array detector (DAD), a temperature-controlled auto sampling unit and temperature-controlled column housing. Agilent ChemStation Software was used for data collection, peak integration and data analysis.

A ThermoFisher Scientific DNAPac Reversed Phase column (Waltham, USA) containing $4 \mu \mathrm{m}$ polymeric particles with proprietary wide pore sizing was used (Dimensions: $2.1 \times 100 \mathrm{~mm}$ ).

\section{Method}

All chromatograms were recorded at a wavelength of 260 $\mathrm{nm}$. Separations were carried out using a sample injection volume of $4 \mu \mathrm{L}$.

The mobile phases were prepared by dissolving the alkylamine into acetonitrile at the selected concentration before the addition of deionised water. When using TEAA, the mobile phase A did not contain any MeCN. Consequently, the TEA was added directly into the deionised water. Mobile phases were produced at selected alkylamine concentrations - $15 \mathrm{mM}, 25 \mathrm{mM}, 100 \mathrm{mM}$ for hexylamine, dibutylamine and triethylamine respectively. Acetate ion pair combinations were formed by adjusting the mobile phase $\mathrm{pH} 8.5$ by titration, through addition of glacial acetic acid. Mobile phase compositions and gradients are detailed in full within the supplementary material (Table 1 and 2). Mobile phases not in use were stored in the refrigerator at $5^{\circ} \mathrm{C}$

An analysis time window for elution of the RNA ladder was set at 15 minutes. A target retention time for the 100nucleotide peak was set at 3 minutes, while for the 1000nucleotide peak it was set to 14 minutes. Optimal mobile phase flow rate was $0.2 \mathrm{~mL} / \mathrm{min}$, while column temperature was $80^{\circ} \mathrm{C}$.

For initial tests, a steep mobile phase gradient was utilised in order to evaluate an approximate range for RNA ladder 
sample retention. The mobile phase gradient could then be adjusted to meet the analysis window criteria. Typically, 3 blank injections were performed before any sample injections in order to allow the column to reach equilibrium. Analysis was performed on the eGFP mRNA sample using the optimal gradient to determine whether any further adjustment was required.

For the optimized method, utilising the DNAPac column $\left(0.2 \mathrm{~mL} / \mathrm{min}\right.$ flow rate at $\left.80^{\circ} \mathrm{C}\right)$, the mobile Phase A consisted of $100 \mathrm{mM}$ TEA in $\mathrm{H} 2 \mathrm{O}(\mathrm{pH} 8.5)$ and mobile Phase B consisted of $100 \mathrm{mM}$ TEA in $40 \% \mathrm{MeCN}$ (pH 8.5). Elution within the analysis time window was achieved with 8.5-12.5\% MeCN in $15 \mathrm{~min}, 12.5-8.5 \% \mathrm{MeCN}$ in $1 \mathrm{~min}$ and $8.5 \% \mathrm{MeCN}$ for 4 minutes. Total run time was 20 minutes.

Half peak height method was used to determine resolution values for the fragment sizes of interest. Details of resolution calculation method can be found in the supplementary material (Equations 1-6).

\section{Results and Discussion}

\section{Model Compounds}

In this study, we evaluate the performance of three alkylamines, commonly used for separation of large RNA, in combination with acetate, as potential ion pairing agents for a stability indicating method for modified mRNA. During initial method development, separations using an RNA ladder, with fragment sizes ranging from 100-1000 nucleotides, were performed to evaluate key separation parameters. The largest fragments in the RNA ladder were 750 and 1000 nucleotides respectively. We aimed to maximise the resolution between these fragments to improve method performance for eGFP modified mRNA (996 nucleotides). Examination of longer mRNA in addition to other modification types would be of interest in future work.

\section{Development of a stability indicating method Method Run Time}

The short method run time of 15 minutes was deemed to be an essential criteria for enabling reliable stability indication, given that mRNA can be susceptible to rapid degradation under certain conditions. Separation of all fragment sizes across the RNA ladder range, within the chosen analysis time window, enables visualisation of changing chromatographic parameters on the separation between fragments. In addition, degradation products resulting from the mRNA do not have a defined size length. Therefore, prior optimization of the method parameters on a similarly sized RNA ladder supports the accurate quantification of degradation products from the mRNA during stress testing.

\section{Column / Stationary Phase}

Kanavarioti has previously demonstrated high resolution separations using the wide-pore DNAPac RP column for the analysis of large RNA using reversed phase ion-pair chromatography ${ }^{22}$. Consequently, we also selected this column to perform our method development process.

\section{Effect of Column Temperature on RNA Separation}

To assess the effect of column temperature on ion pair separations of large RNA, we performed RNA marker separations at both $50^{\circ} \mathrm{C}$ and $80^{\circ} \mathrm{C}$ with $100 \mathrm{mM}$ TEA, using an identical gradient (Supplementary Material Figure 1). The gradient slope was optimised for $80^{\circ} \mathrm{C}$, with both separations conducted within the set 15 -minute analysis time window. At increased column temperature, we observed improved resolution between the 750/1000 nucleotide fragments. At $50^{\circ} \mathrm{C}$ and $80^{\circ} \mathrm{C}$, the chromatographic resolution of these fragments were calculated to be 1.9 and 2.2 respectively. Higher column temperature also contributed to slightly improved peak shapes across the marker size range. An additional benefit from operation at a higher column temperature was a small reduction in overall analysis time. As high temperature column conditions did not appear to impact the stability of the analyte, we opted to fix the column temperature at $80^{\circ} \mathrm{C}$ throughout the method development process.

\section{Effect of Mobile Phase Flow Rate on RNA Separation}

The effect of mobile phase flow rate on the analyses was examined by performing RNA marker separations at $80^{\circ} \mathrm{C}$, using $100 \mathrm{mM}$ TEA, under different column flow rates with a fixed gradient (Supplementary Material Figure 2). It was observed that mobile phase flow rates above $0.2 \mathrm{~mL} / \mathrm{min}$ resulted in a significant decrease to the heights of the 750 and 1000 nucleotide fragments peaks (Supplementary Material Table 3). To date there are very few studies describing the retention mechanism of large RNA in IP-RPLC, especially in relation to the effect of flow rate on the obtained peak height and area. In practice, however, decreased peak heights may limit the usability of the method for accurate quantification any degradation products similar to that in size of the mRNA main peak.

At mobile phase flowrates above $0.2 \mathrm{ml} / \mathrm{min}$, retention of the 100 nucleotide fragment peak was shown to be less robust. Limited separation was achievable between the system peak and the 100-nucleotide fragment peak. Negligible separation between these points on the chromatogram would limit quantification of any RNA fragments shorter than 100 nucleotides. This was a characteristic that was not deemed advantageous for the stability determination of modified mRNA compounds, where degradation products could potentially fall in this 0-100 nucleotide range.

Interestingly, the highest calculated resolution value, between the 750/1000 nucleotide fragments, using the fixed mobile phase gradient was measured at a mobile phase flow rate of $0.6 \mathrm{~mL} / \mathrm{min}$ (Supplementary Material Table 4). However, operation above $0.2 \mathrm{~mL} / \mathrm{min}$ had a significant adverse impact on RNA peak shapes and peak separation capability. Optimal flow rate for this method was found to be a compromise between achieving higher resolution values for the fragment sizes of interest, peak heights that would enable accurate quantification of large sample fragments, and efficient separation between 
the earlier eluting peaks. A constant flow rate of 0.2 $\mathrm{mL} / \mathrm{min}$ was found to be most suitable for all sample analyses using the DNAPac RP column. Potentially there is an opportunity to enable more informative separation analyses of large RNA through further manipulation of the mobile phase flow rate. This would be an aim for an additional investigation and could include modulation of mobile phase flow rates across a single analysis run.

\section{Evaluation of Ion Pairing Agents}

Several parameters related to the ion-pairing mechanism can affect the chromatographic retention of RNA, including alkylamine concentration, structure and solubility as well as the mobile phase $\mathrm{pH}$. The interplay between these parameters can make method development challenging. Comparison between different ion-pairing agents are typically a compromise between which parameters one selects to optimise.

In this study, we have attempted to perform representative best-case separations for each of the selected IPs, using only linear gradients. Step wise linear gradients have previously been used to separate both small and large RNA fragments ${ }^{13,15}$. However, as part of our evaluation strategy, linear gradients enabled us to limit the number of parameters to test and optimise. All alkylamine concentrations were fixed to proposed optimal values as reported in the literature (Table 1) ${ }^{30-32}$.

Table 1: Proposed Optimal Mobile Phase Alkylamine Concentrations

\begin{tabular}{ll}
\hline Alkylamine & $\begin{array}{l}\text { Concentration } \\
(\mathrm{mM})\end{array}$ \\
\hline Hexylamine & 15 \\
Dibutylamine & 25 \\
Triethylamine & 100 \\
\hline
\end{tabular}

All mobile phases were adjusted to $\mathrm{pH} 8.5$ through the addition of glacial acetic acid. This $\mathrm{pH}$ value was selected as it is well below that of the pKa values for the chosen alkylamines, thus ensuring complete ionisation within the mobile phases ${ }^{32}$. Additionally, $\mathrm{pH} 8.5$ was not shown to impact the stability of the model RNA compounds during analysis.

During our method development process, we observed that both gradient slope and $\mathrm{MeCN}$ starting concentration had to be optimised for each IP combination to enable elution within the set analysis time for the RNA marker sample. Starting MeCN concentrations for both the HAA and the DBAA were approximately similar, $32.3 \%$ and $34.6 \%$ respectively, however, when using DBAA a steeper gradient was required $\left(0.80 \% \mathrm{~min}^{-1} \mathrm{MeCN}\right)$ in order to achieve the desired elution profile. Comparatively, HAA required a slightly flatter gradient $\left(0.53 \% \mathrm{~min}^{-1} \mathrm{MeCN}\right)$. The RNA marker sample was found to be less well retained on the DNAPac column, using TEAA, when compared to the other two IP agents at the selected concentrations. Both a lower starting $\mathrm{MeCN}$ concentration $(8.6 \%)$ and flatter gradient $(0.27 \%$ $\min ^{-1} \mathrm{MeCN}$ ) were used in order to obtain separations within the 15 minute analysis time window.

Under the respective chromatographic conditions, full separation of the RNA marker fragments was achieved, using each of the selected ion pairs, within the 15 minute analysis window. The baselines achieved in each of the analyses were shown to be both stable and reproducible across repeated runs. As RNA size length increases, we observed it becomes more challenging to separate similarly sized fragments from one another (Figure 1). This has previously been noted for IP-RPLC analyses as well as using orthogonal separation techniques for RNA, such as capillary gel electrophoresis ${ }^{15}, 33$. The lower separation power for the larger RNA will influence the development strategy for a stability indicating method. In these cases, the method optimisation is a significant challenge and non-linear/step-wise gradients in combination with longer analysis times could offer opportunity to improve separation performance.
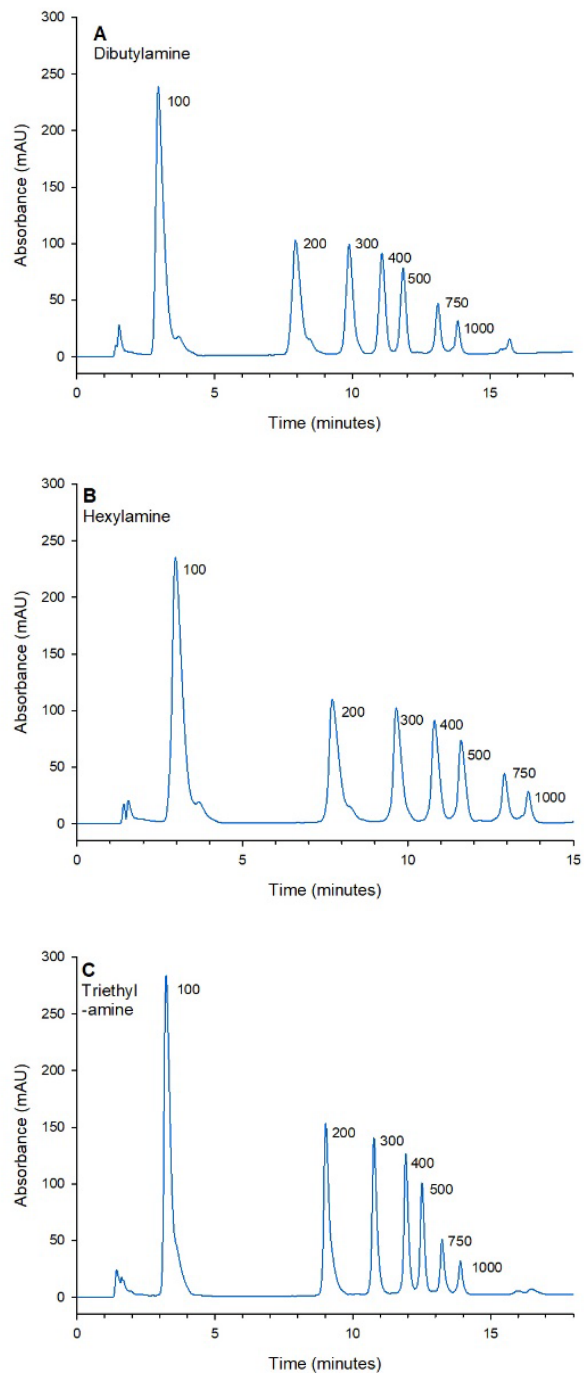

Figure 1: Comparison of different ion pair agents for the separation of a single stranded RNA ladder 100-1000 nucleotides using the polymer based DNAPac RP column.

(A) $25 \mathrm{mM}$ DBA $(34.6 \%-46.6 \% \mathrm{MeCN})$ (B) $15 \mathrm{mMHA} \quad(32.3 \%$ $40.3 \% \mathrm{MeCN})(\mathrm{C}) 100 \mathrm{mM}$ TEA $(8.6 \%-12.6 \% \mathrm{MeCN})$ 
Separations performed using HAA and DBAA, resulted in similar peak patterns and retention times at the chosen alkylamine concentrations. However, comparison of the calculated resolution values showed DBAA offered improved separation power when compared to the HAA for the larger fragments sizes - most similar in size to that of the model mRNA (Table 2). The highest resolution was achieved using TEAA, which enabled a resolution of 2.5 to be obtained between the 750 and 1000 nucleotide RNA marker fragments. In samples analysed using both HAA and DBAA, it was possible to detect impurities alongside the 100 and 200 nucleotide fragment peaks (Figure $1 \mathrm{~A} / \mathrm{B}$ ). However, in analysis using TEAA, there appears to be less resolving power for the shorter fragments, as the impurities seem to co-elute with the 100 and 200 nucleotide peaks. This highlights the potential opportunity to combine ion-pairs for improved separation performance across a wider fragment size range.

Table 2: Resolution Values Between 750/1000 Nucleotide RNA Ladder Sample Fragments for Selected Alkylamines

\begin{tabular}{ll}
\hline Alkylamine & Resolution \\
\hline Hexylamine & 2.1 \\
Dibutylamine & 2.3 \\
Triethylamine & 2.5 \\
\hline
\end{tabular}

The method parameters developed for the RNA ladder sample, using each of the selected alkylamines, were subsequently applied to analyse the model mRNA compound - modified eGFP mRNA (996 nucleotides) (Figure 2). When utilising DBAA and HAA, retention times for the main mRNA peak corresponded to that of the 1000 nucleotide sized fragment from the RNA marker sample. However, the peak shapes using these ion-pair agents were not optimal for the eGFP mRNA - displaying a broader main peak (Table 3).
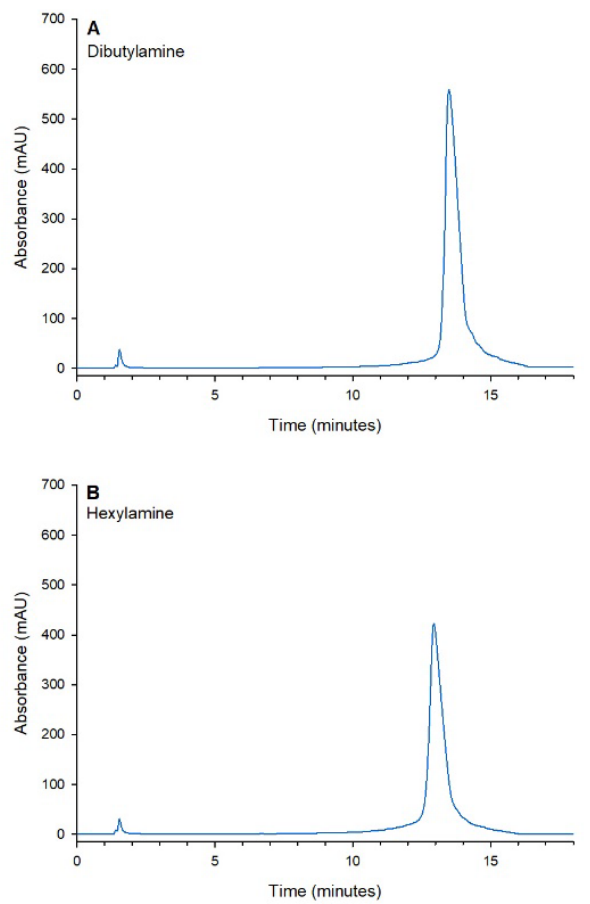

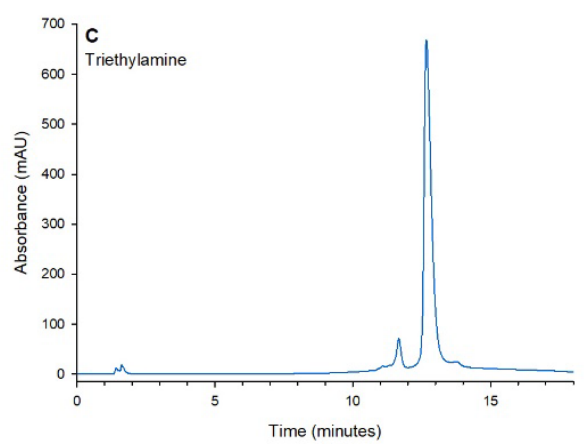

Figure 2: Comparison of different ion pair agents for the separation of eGFP mRNA with 996 nucleotides using the polymer based DNAPac RP column. (A) $25 \mathrm{mM}$ DBA $(34.6 \%-46.6 \% \mathrm{MeCN})$ (B) $15 \mathrm{mM}$ HA $(32.3 \%-40.3 \% \mathrm{MeCN})(\mathrm{C}) 100 \mathrm{mM}$ TEA $(8.6 \%-$ $13.7 \% \mathrm{MeCN})$

Table 3: eGFP mRNA Main Peak Half Height Width Using the Selected Alkylamines (Optimised Method Parameters Within 15 Minute Analysis Time Window)

\begin{tabular}{ll}
\hline Alkylamine & $\begin{array}{l}\text { eGFP mRNA Half } \\
\text { Height Peak Width } \\
\text { (Minutes) }\end{array}$ \\
\hline Hexylamine & 0.51 \\
Dibutylamine & 0.57 \\
Triethylamine & 0.27 \\
\hline
\end{tabular}

This would likely impact quantification of any longmer/shortmer impurities similar in size to the intact mRNA, potentially limiting the usability of the method for compounds of this type.

Using TEAA, the gradient endpoint was adjusted to $13.5 \% \mathrm{MeCN}\left(0.33 \% \mathrm{~min}^{-1} \mathrm{MeCN}\right)$ to ensure that the eGFP mRNA eluted within the 15 minute analysis time window. As a consequence of this adjusted gradient, a slightly narrower mRNA main peak is observed (Supplementary Material Figure 3). Additionally, there is a separated pre-main eGFP peak. This was also reported by Kanavarioti for eGFP mRNA ${ }^{22}$. The lack of pre-main peak implies that DBAA and HAA were both unable to resolve the main peak from pre-existing shortmer fragments at the applied separation parameters. The improved separation performance for the eGFP mRNA, using TEAA, is concurrent with the superior resolution that was obtained between the largest sized fragments of the RNA ladder sample, using this ion-pair.

Close et al have previously reported separation of RNA fragments based on both size and base composition when using TEAA. They propose that short chain ion-pairing agents, such as TEAA, only partially cover the stationary phase and may in fact allow direct reversed phase interactions between the RNA strands and the stationary phase ${ }^{15}$. The shift in retention time between the 1000nucleotide RNA fragment, in the ladder sample, and the similarly sized eGFP mRNA, could therefore be a product of differing structure/base composition between these two analytes. This was an important finding, showing that the method developed for the RNA ladder sample was not directly applicable for analysis of the eGFP mRNA. We 
propose that separation methods, for other mRNAs, can be developed using a similar approach as described above. However, the method parameters may require additional optimisation adjusting to the size and sequence of the analyte. Analysis of eGFP mRNA separations carried out in triplicate, under our newly optimised method conditions using $100 \mathrm{mM}$ TEA in the mobile phase displayed excellent repeatability (Supplementary Material Figure 4).

\section{Evaluation of Method Stability Indicating Power}

As TEAA was shown to offer superior separation capabilities for the larger RNA fragments, applying our method parameters, we selected this ion-pair agent to evaluate the stability indicating power of our IP-RPLC method for the selected eGFP modified mRNA.

To our knowledge, there are few reports about the major degradation pathways and resulting degradation products with respect to pharmaceutical development of mRNA-based therapeutics. Stress testing conditions selected were exposure to heat, hydrolytic conditions, and addition of ribonucleases. The potential degradation products could comprise of fragments similar in size to the main component, down to single nucleotide fragments . Applying our optimised LC method, using 100mM TEA, we expected to observe decreased main peak area as well as an increased percentage of pre-main peak area and increased number of smaller fragment peaks.

Stress inducing hydrolytic conditions applied directly to the mRNA sample were shown to result in rapid degradation, visible as an increase in the pre-main peak areas (Figure 3). Hydrolysis of the model mRNA, at both high and low $\mathrm{pH}$, took place after a short exposure time. For both samples, the main peak height decreased by more than $50 \%$ within 30 minutes of sample preparation, indicating that the mRNA was unstable under both sets of conditions (Figure 3B/3C). Decrease in main peak height however, is more pronounced in the acidic conditions, in comparison to the basic environment. Under both sets of conditions, the degradation products from hydrolysis appear in the same retention time window as the existing impurities in the untreated mRNA.

Exposure to the RNase A, at the chosen concentration, resulted in a more prolonged degradation process extending to over 12 hours. The RNase A degradation pathway appears markedly different to that of the other stress tested conditions, with the pre-main peak appearing far less prominently (Figure 3D). More notably however, at similar main peak heights, the earlier eluting peak (2 minutes) is significantly larger than in either hydrolysis analyses, suggesting the degraded fragments break down to smaller less well retained fragments more readily. This is to be expected as the enzyme breaks the polynucleotide chain at specific and defined intervals, through endonuclease cleavage. The distinctive peak pattern observed is therefore likely related to the mRNA base composition and structure, when compared to the non- specific hydrolysis degradation. A blank run of 0.005 $\mathrm{ng} / \mu \mathrm{L}$ RNase A solution without RNA, confirmed that the earlier eluting peak corresponded only to smaller less well/unretained RNA fragments rather than any RNase A residues.
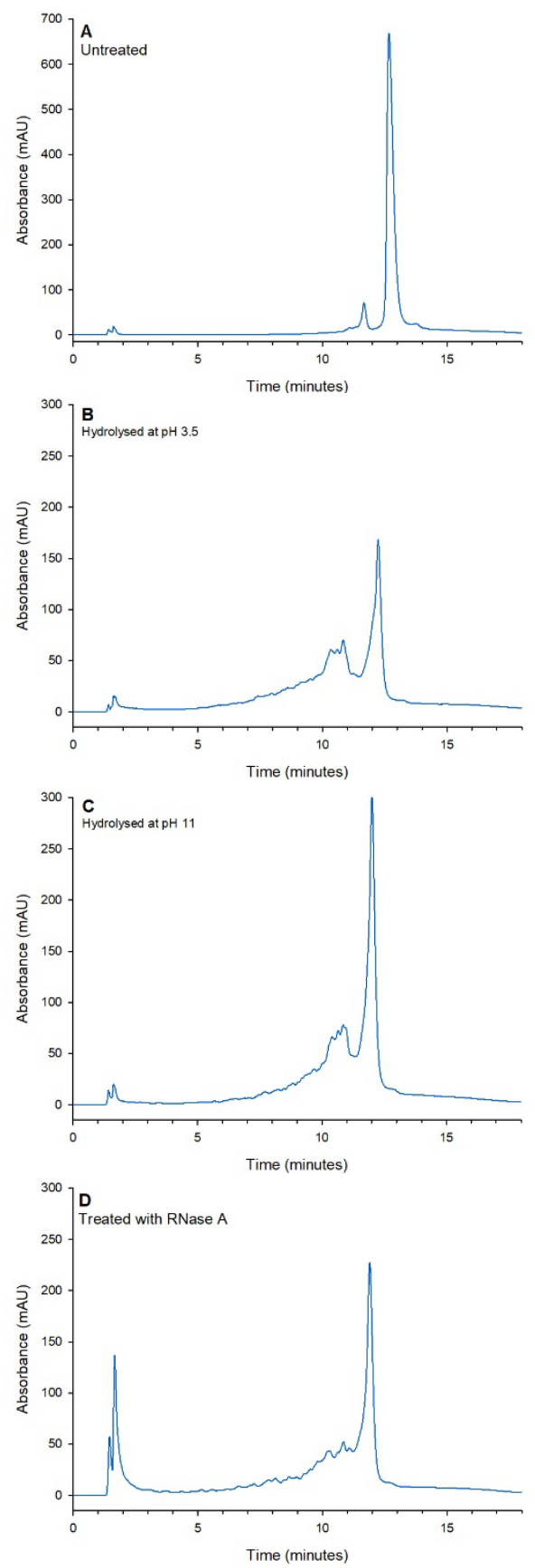

Figure 3: Analysis of the stress-tested model compound, modified eGFP mRNA (996 nucleotides), using optimized method conditions. Separation with DNAPac RP column with TEAA in the mobile phase $(8.6 \%-13.7 \% \mathrm{MeCN})$. Further experimental details are provided in the Experimental Section. (A) Untreated eGFP mRNA (B) Hydrolysis at $\mathrm{pH}=3.5,30 \mathrm{~min}$ after preparation, (C) Hydrolysis at $\mathrm{pH}=11,30$ min after preparation (D) Exposed to RNase A, 12 hours after preparation.

Degradation through exposure of the model mRNA to elevated temperatures could also be well visualised using our optimised IP-RPLC method (Figure 4). A reduction in percentage main peak area is observed with increasing 
sample exposure time to high temperature conditions (Table 4).
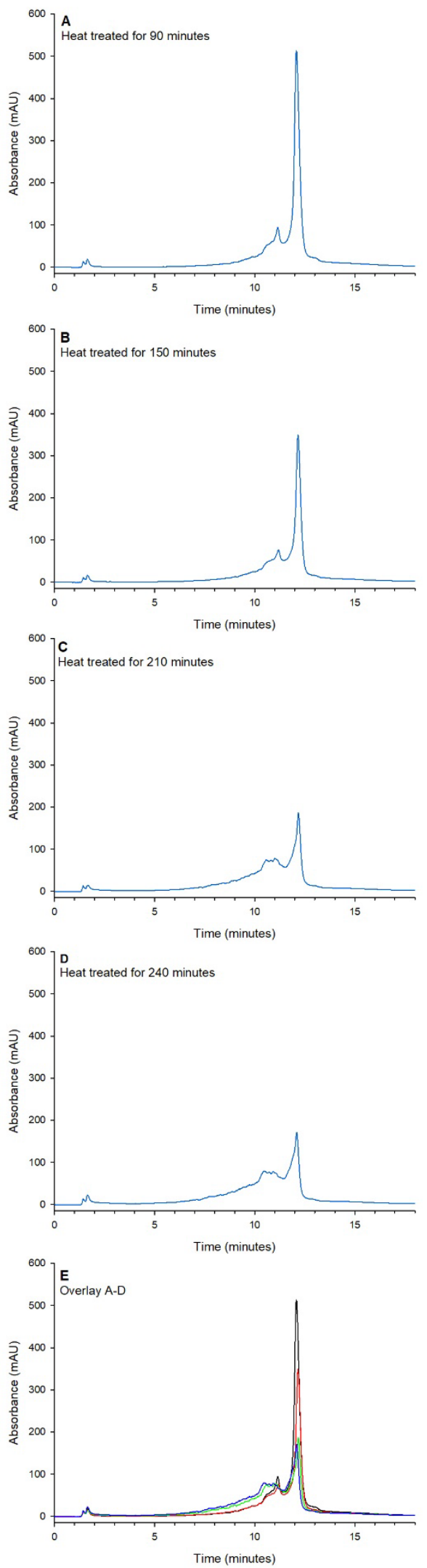

Figure 4: Analysis of the heat-treated model compound, modified eGFP mRNA ( 996 nucleotides), at $85^{\circ} \mathrm{C}$ using optimized method conditions. Separation with DNAPac RP column with TEAA in the mobile phase $(8.6 \%-13.7 \% \mathrm{MeCN})$. Further experimental details are provided in the Experimental Section. (A) Sample heat treated $90 \mathrm{~min}$ at $85^{\circ} \mathrm{C}$ (B) Sample heat treated $150 \mathrm{~min}$ at $85^{\circ} \mathrm{C}$, (C) Sample heat treated $210 \mathrm{~min}$ at $85^{\circ} \mathrm{C}$ (D) Sample heat treated $240 \mathrm{~min}$ at $85^{\circ} \mathrm{C}$ and (E) Overlay of sample heat treated $90,150,210$ and $240 \mathrm{~min}$ at $85^{\circ} \mathrm{C}$.

Table 4: eGFP mRNA Main Peak and Pre-Main Peak Percentage Areas Upon Increasing Exposure Time at $85^{\circ} \mathrm{C}$

\begin{tabular}{lll}
$\begin{array}{l}\text { Sample Exposure } \\
\text { Time } \\
\text { at } 85^{\circ} \mathrm{C} \text { (Minutes) }\end{array}$ & $\begin{array}{l}\text { Percentage Main } \\
\text { Peak Area } \\
(\%)\end{array}$ & $\begin{array}{l}\text { Percentage Pre- } \\
\text { Main Peak Area } \\
(\%)\end{array}$ \\
\hline 15 & 85 & 15 \\
30 & 85 & 15 \\
90 & 69 & 31 \\
150 & 66 & 34 \\
210 & 42 & 58 \\
240 & 36 & 64 \\
\hline
\end{tabular}

Sample exposed for 15 minutes at $85^{\circ} \mathrm{C}$ did not appear to show any heat induced degradation effects (Supplementary Material Figure 5A). This confirms that high temperature column conditions should not impact the stability of the eGFP mRNA, using our analysis method. Moreover, only minor temperature-induced mRNA degradation can be observed after 30 minutes sample exposure time, at $85^{\circ} \mathrm{C}$ (Supplementary Material Figure 5B). These effects start to become more pronounced after 90 minutes exposure time. This would suggest that the modified mRNA was relatively robust upon exposure to high temperature conditions for short to medium length temperature excursions.

However, it should be noted that the stress conditions applied may also have an effect on the analyte's conformational structure and thus its resulting chromatographic behaviour. We observed that treatment of the sample under high temperature conditions resulted in small changes of the post main peak area (Supplementary Figure 6). The main peak retention time remains consistent throughout analyses, providing indication that limited conformational changes take place (Figure 4E).

\section{Conclusion}

In this study, a robust stability indicating IP-RPLC method has been successfully developed for modified eGFP mRNA. Through evaluation of key chromatographic parameters the method was optimised to enable high resolution separations of large RNA fragments sized up to 1000 nucleotides. Use of high column temperature was shown to deliver improved peak resolution during the separation analyses. In addition, we found that the mobile phase flow rate was important in controlling the elution profile of the RNA analytes. All of our chosen ion-pairing agents were successfully able to baseline separate the fragments in our chosen RNA ladder sample, during a 15 minute analysis time window. However, we found using $100 \mathrm{mM}$ Triethylamine in the mobile phase offered better separation power for the largest RNA sample fragments, as well as for the eGFP modified mRNA. A key finding was that the optimised method could be used to study the degradation of the 
mRNA under several stress-inducing conditions (heat, hydrolytic conditions and exposure to ribonucleases). Our results showed that quantification of the amount of degradation products eluting before the mRNA main peak could be performed and the relative stability of the mRNA analyte determined. To the best of our knowledge, this is the first study using a wide set of stress testing conditions relevant for pharmaceutical development of an mRNA drug product. The methodology discussed within this work can therefore be used to guide method development strategies for the assessment of mRNA drug product/substance stability.

\section{Associated Content}

\section{Supplementary Information}

The supplementary material contains tables of mobile phase compositions and method conditions for each analysis run, equations detailing the resolution calculation method and supporting chromatographs and tables.

\section{Author Contributions}

Jonathan Currie: Conceptualisation, Methodology, Formal Analysis, Investigation, Writing Original Draft, Writing - Review \&

Editing and Visualisation.

Jacob R. Dahlberg: Conceptualisation, Methodology, Formal Analysis, Investigation, Writing Original Draft and Writing - Review \& Editing.

Jonas Eriksson: Conceptualisation and Writing Review \& Editing.

Fritz Schweikart: Conceptualisation, Methodology, Formal Analysis and Writing - Review \& Editing.

Gunilla A. Nilsson: Conceptualisation and Writing Review \& Editing.

Eivor Örnskov: Conceptualisation, Methodology, Formal Analysis, Investigation, Writing Original Draft, Writing - Review \& Editing,

Visualisation and Supervision.

\section{Acknowledgements}

The authors would like to thank, Nadim Akhtar, Anders Karlsson, Linda Thunberg and Stephen Wren, from AstraZeneca, for valuable discussions regarding the work and their assistance in reviewing the manuscript. This research did not receive any specific grant from funding agencies in the public, commercial, or not-forprofit sectors.

\section{References}

1. Ashford, M., Drug delivery - the increasing momentum. Drug Delivery and Translational Research 2020, 10, 1888-1894.

2. Van Hoecke, L.; Roose, K., How mRNA therapeutics are entering the monoclonal antibody field. Journal of Translational Medicine 2019, 17 (1), 1-14. 3. Gan, L. M.; Lagerström-Fermér, M.; Carlsson, L. G.; Arfvidsson, C.; Egnell, A. C.; Rudvik, A.; Kjaer, M.; Collén, A.; Thompson, J. D.; Joyal, J.; Chialda, L.; Koernicke, T.; Fuhr, R.; Chien, K. R.; FritscheDanielson, R., Intradermal delivery of modified mRNA encoding VEGF-A in patients with type 2 diabetes. Nature Communications 2019, 10 (1), 871-880.

4. Wang, F.; Zuroske, T.; Watts, J. K., RNA therapeutics on the rise. Nature Reviews Drug Discovery 2020, 19, 441-442.

5. Delivering the promise of RNA therapeutics. Nature Medicine 2019, 25 (9), 1321-1321.

6. Kulkarni, J. A.; Witzigmann, D.; Thomson, S. B.; Chen, S.; Leavitt, B. R.; Cullis, P. R.; van der Meel, R., The current landscape of nucleic acid therapeutics. Nature Nanotechnology 2021, 16 (6), 630-643.

7. Sahin, U.; Kariko, K.; Tuereci, O., mRNAbased therapeutics - developing a new class of drugs. Nature Reviews Drug Discovery 2014, 13 (10), 759-780.

8. Note For Guidance on Stability Testing: Stability Testing of New Drug Substances and Products. In (CPMP/ICH/2736/99), EMA, Ed. European Medicines Agency: http://www.emea.eu.int, 2003; p 20.

9. (CBER), C. f. B. E. a. R., Guidance for Industry

Content and Format of Chemistry, Manufacturing and Controls Information and Establishment Description Information for a Vaccine or Related Product. FDA, Ed. 1999; p 30.

10. Hinz, T. In Regulation of RNA-Based Medicines, 3rd International mRNA Health Conference, Berlin, Germany, 11-12 November 2015; Berlin, Germany, 2015. 11. Yamauchi, Y.; Taoka, M.; Nobe, Y.; Izumikawa, K.; Takahashi, N.; Nakayama, H.; Isobe, T., Denaturing reversed phase liquid chromatographic separation of non-coding ribonucleic acids on macroporous polystyrene-divinylbenzene resins. Journal of Chromatography A 2013, 1312, 87-92.

12. Huang, Z.; Jayaseelan, S.; Hebert, J.; Seo, H.; Niu, L., Single-nucleotide resolution of RNAs up to 59 nucleotides by high-performance liquid chromatography. Analytical Biochemistry 2013, 435 (1), 35-43.

13. Azarani, A.; Hecker, K. H., RNA analysis by ion-pair reversed-phase high performance liquid chromatography. Nucleic Acids Research 2001, 29 (2), 19.

14. Dickman, M. J., Ion Pair Reverse-Phase Chromatography: A Versatile Platform for the Analysis of RNA. Chromatography Today 2011, March 2011, 2226.

15. Close, E. D.; Nwokeoji, A. O.; Milton, D.; Cook, K.; Hindocha, D. M.; Hook, E. C.; Wood, H.; Dickman, M. J., Nucleic acid separations using superficially porous silica particles. Journal of Chromatography A 2016, 1440, 135-144.

16. Georgopoulos, D. E.; Leibowitz, M. J., Use of high-performance liquid chromatographic fractionation of large RNA molecules in the assay of group I intron ribozyme activity. Journal of Chromatography A $\mathbf{2 0 0 0}$, 868 (1), 109-114.

17. Gjerde, D. T.; Hoang, L.; Hornby, D., RNA

Purification and Analysis: Sample Preparation,

Extraction, Chromatography. first ed.; Wiley-VCH Verlag: Weinheim, 2009.

18. Lloyd, L. L.; Millichip, M. I.; Mapp, K. J., Rigid polymerics: the future of oligonucleotide analysis 
and purification. Journal of Chromatography A 2003, 1009 (1), 223-230.

19. Waghmare, S. P.; Pousinis, P.; Hornby, D. P.; Dickman, M. J., Studying the mechanism of RNA separations using RNA chromatography and its application in the analysis of ribosomal RNA and RNA:RNA interactions. Journal of Chromatography $A$ 2009, 1216 (9), 1377-1382.

20. Roussis, S. G.; Pearce, M.; Rentel, C., Small alkyl amines as ion-pair reagents for the separation of positional isomers of impurities in phosphate diester oligonucleotides. Journal of Chromatography A 2019, 1594, 105-111.

21. Li, L.; Foley, J. P.; Helmy, R., Simultaneous separation of small interfering RNA and lipids using ionpair reversed-phase liquid chromatography. Journal of Chromatography A 2019, 1601, 145-154.

22. Kanavarioti, A., HPLC methods for purity evaluation of man-made single-stranded RNAs. Scientific Reports 2019, 9 (1), 1019-1032.

23. Jiang, T.; Yu, N.; Kim, J.; Murgo, J.-R.; Kissai, M.; Ravichandran, K.; Miracco, E. J.; Presnyak, V.; Hua, S., Oligonucleotide Sequence Mapping of Large Therapeutic mRNAs via Parallel Ribonuclease Digestions and LC-MS/MS. Analytical Chemistry 2019, 91 (13), 8500-8506.

24. Nwokeoji, A. O.; Earll, M. E.; Kilby, P. M.; Portwood, D. E.; Dickman, M. J., High resolution fingerprinting of single and double-stranded RNA using ion-pair reverse-phase chromatography. Journal of Chromatography. B, Analytical Technologies in the Biomedical and Life Sciences 2019, 1104, 212-219. 25. Nunomura, A.; Lee, H.-g.; Zhu, X.; Perry, G., Consequences of RNA oxidation on protein synthesis rate and fidelity: implications for the pathophysiology of neuropsychiatric disorders. Biochemical Society Transactions 2017, 1053-1066.

26. Simms, C. L.; Zaher, H. S., Quality control of chemically damaged RNA. Cellular and Molecular Life Sciences 2016, 73 (19), 3639-3653.

27. Alseth, I.; Dalhus, B.; Bjørås, M., Inosine in DNA and RNA. Current Opinion in Genetics \& Development 2014, 26, 116-123.

28. An, R.; Jia, Y.; Wan, B.; Zhang, Y.; Dong, P.; Li, J.; Liang, X.; Bhaumik, S. R., Non-Enzymatic Depurination of Nucleic Acids: Factors and Mechanisms. PLoS ONE 2014, 9 (12), 1-17.

29. Schoenmaker, L.; Witzigmann, D.; Kulkarni, J. A.; Verbeke, R.; Kersten, G.; Jiskoot, W.; Crommelin, D. J. A., mRNA-lipid nanoparticle COVID-19 vaccines: Structure and stability. International Journal of

Pharmaceutics 2021, 601, 120586.

30. Goyon, A.; Yehl, P.; Zhang, K., Characterization of therapeutic oligonucleotides by liquid chromatography. Journal of Pharmaceutical and Biomedical Analysis 2020, 182, 1-13.

31. Li, N.; El Zahar, N. M.; Saad, J. G.; van der Hage, E. R. E.; Bartlett, M. G., Alkylamine ion-pairing reagents and the chromatographic separation of oligonucleotides. Journal of Chromatography A 2018, 1580, 110-119.
32. Gong, L., Comparing ion-pairing reagents and counter anions for ion-pair reversed-phase liquid chromatography/electrospray ionization mass spectrometry analysis of synthetic oligonucleotides. Rapid Communications in Mass Spectrometry 2015, 29 (24), 2402-2410.

33. Lu, T.; Klein, L. J.; Ha, S.; Rustandi, R. R., High-Resolution capillary electrophoresis separation of large RNA under non-aqueous conditions. Journal of Chromatography A 2020, 1618, 1-7. 